\title{
A rare case of takotsubo syndrome within the first day after heart transplantation
}

\author{
Mohammed Al Humaid ${ }^{1}$, Mawada Ahmed², Domenico Galzerano ${ }^{3}$, Mosaad Al Hussein², \\ Mohammed Al Admawi², Jehad Al Buraiki², Hani Alsergani², Olga Vriz ${ }^{2}$ \\ ${ }^{1}$ Cardiology Center, King Saud Medical City, Riyadh; ${ }^{2}$ Heart Centre Department, King Faisal Specialist Hospital \& \\ Research Center, Riyadh; ${ }^{3}$ College of Medicine, Alfaisal University, Riyadh, Saudi Arabia
}

\begin{abstract}
A 39-year-old woman underwent heart transplantation (HTx) for advanced heart failure. The donor was a 36-year-old young woman without past medical history. The first day after HTx, Twaves changes were noted. Echocardiography revealed akinesia/dyskinesia of all basal segments of the two ventricles. Coronary catheterization plus biopsy were done 7 days later showing no coronary obstruction, no rejection and complete recovery of wall motion abnormalities on echocardiogram, suggesting biventricular inverted takotsubo syndrome (TTS). This is a case of TTS during the first day after HTx, with completely denervated heart but because of the inotropic drug support it still represents a target for catecholamine-induced cardiac dysfunction.
\end{abstract}

\section{Introduction}

Takotsubo syndrome is supposed to be a disturbance of myocardial microcirculation related to increased concentration of

Correspondence: Mohammed Al Humaid, Cardiology Center, King Saud Medical City, Riyadh, Saudi Arabia.

Tel. +966.547000966 - Fax: +966.1.14355555.

E-mail: alhumid1428@hotmail.com

Key words: Heart transplant; Takotsubo; cardiomyopathy.

Contributions: MAHum, MAA, MAHus, data collection; MAHum, OV, manuscript drafting; DG, MAHus, MAAd, JAB, HA, important intellectual content, case report discussion. All authors have participated in the work and have reviewed and agree with the content of the final version.

Conflict of interest: The authors declare no conflict of interest.

Received for publication: 14 August 2019.

Accepted for publication: 4 September 2019.

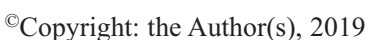

Licensee PAGEPress, Italy

Monaldi Archives for Chest Disease 2019; 89:1149

doi: 10.4081/monaldi.2019.1149

This article is distributed under the terms of the Creative Commons Attribution Noncommercial License (by-nc 4.0) which permits any noncommercial use, distribution, and reproduction in any medium, provided the original author(s) and source are credited. catecholamines (i.e., epinephrine, norepinephrine, and dopamine), and wall motion abnormalities with different involvement of the myocardial muscle and usually followed but complete recovery of contractility $[1,2]$. The most common type is the apical one with apical akinesia of the left ventricle and mid-basal hypercontractility. Catecholamines increase may be related to sympathetic overactivity caused by stress or inotropic drugs.

\section{Case Report}

A 39-year-old woman underwent heart transplantation for New York Heart Association Class IV heart failure symptoms despite guideline-directed medical therapy. She had underlying rheumatic heart disease with mitral valve repair 10 years prior to transplantation. The donor was a 36-year-old female without significant past medical history who was declared brain-dead due to cerebral vascular accident. The donor echocardiogram that was performed $20 \mathrm{~h}$ prior to procurement was normal showing no wall motion abnormalities. On visual inspection during heart procurement the heart function was normal and palpation of the epicardial coronary arteries did not reveal any gross pathology, areas of atheromatous disease, or calcium. The crossmatch was negative.

An orthotopic heart transplant was carried out using the bicaval technique. The surgery was not complicated and the graft function was normal. The patient came off bypass without difficulties and she was admitted to the cardiac surgery intensive care unit intubated and on inotropic drug support. Twelve hours posttransplant, the patient developed new T-wave changes on the monitor. An echocardiogram revealed normal LV size, borderline reduce LV function (ejection fraction $\sim 50 \%$ ), presence of wall motion abnormalities characterized by akinesia of the basal septum and hypokinesia of the other septal segments, dyskinesia of all other basal segments of the LV, akinesia of the basal segment of the right ventricle and hypercontractility of the apical segments of the ventricles. In addition, also the speckle tracking of the two ventricles was significantly compromised. The patient hemodynamics were stable. Coronary angiography plus endomyocardial biopsy was done few days later showing no evidence of coronary atherosclerosis and biopsy result showed mild acute cellular rejection (ISHLT grade 1R) with no findings of antibody mediated rejection. No donor specific antibodies were detected. The echocardiogram performed in the same day showed complete recovery of the wall motion abnormalities while strain was still impaired. A final diagnosis of TTC was therefore made. The third echo done 3 weeks later confirm the complete recovery of wall motion abnormalities, showed a sig- 
nificant improvement in biventricular strain although it was not normal yet (Figures 1 and 2).

\section{Discussion}

The classical clinical presentation of the TTS is similar to acute coronary syndrome (ACS) but with reversible left ventricular dysfunction that is unrelated with obstructive coronary disease $[1,2]$. The pathophysiology seems to be based on excessive local release of catecholamine (i.e., epinephrine, norepinephrine, and dopamine) $[3,4]$ due to sudden stress which starts at the cognitive centers of the brain, follows the hypothalamic-pituitary-adrenal axis (HPA), the thoracic nervous sympathetic system, ending on the cardiovascular apparatus (myocardium, coronary arteries and peripheral vasculature) with microvascular spasm and myocyte injury [5]. In the transplanted hearts, the heart is totally denervated in the acute phase and the stimulation of the cardiomyocyte by catecholamines has to be different from the release of norepinephine in the synaptic cleft by the thoracic sympathetic ganglia [6]. In addition, the transplanted heart has also lost inhibitory parasympathetic innervations which contribute to the exaggerated response to catecholamines. As a consequence, transplanted hearts seem to be more susceptible to non-physiologic doses of exogenous catecholamines such as dopamine, noradrenaline and adrenaline.

To the best of our knowledge this is the first report of TTS, with inverted and biventricular involvement of the heart beyond a single coronary artery territory, in a young woman 24-hour post HTx.

Upon literature review, there are 6 case reports of TTS peri or post HTx (Table 1). Chinali et al. [7] reported a case of TTS following angry debate in a 21-year-old woman with HTx done 10 years back. The patient complained reduced tolerance to physical activity and fatigue. Behnes et al. [8] reported the case of a 64-year-old man with HTx done 9 years earlier. Few days after an acute coronary syndrome (ACS), the patient developed sudden dyspnea. Another case [9] was a 69-year-old man who had an orthotopic HTx 6 years back who presented respiratory distress and hypotension $30 \mathrm{~min}$ after total shoulder arthroplasty. In all of these cases the clinical scenario was dyspnea and congestive heart failure but not chest pain where the most likely explanation is that the sympathetic re-innervation had already occurred, usually happens within the first years, while the nociceptive sensory afferences probably had not. In addition, Gastwirth et al. [10] reported a case of a 55-year-old woman who had HTx 1 year early from a 19-year-old donor who had a dobutamine stress echocardiography and at peak stress and during recovery, images showed significant wall motion abnormalities and severe left ventricular dysfunction which normalized within 5 days. The patient remained always asymptomatic. The authors hypothesized that the high dose of dobutamine on the denervated heart could be the cause. The use of donors with TTS have been reported in the literature. Redfors et al. [11] reported the case of a patient with subarachnoid hemorrhage, suitable for organ donation. Pre HTx coronary angiography revealed normal coronary arteries but midventricular akinesia with reduced EF confirmed by echocardiography. The diagnosis of TTS was made and the heart was used for transplant. One month after HTx, the patient's left ventricular function was normal. The authors hypothesized that the process of brain death could have elicited significant somatic stress and a catecholamine surge. A second case [12] described an apical left ventricular ballooning in the donor's cardiac heart that was probably affected by the intense physiologic stress caused by the fatal motor vehicle accident.

In our case we hypothesize, since the postganglionic sympathetic nerve fibers to the heart were excluded, TTS was caused by the release of catecholamines from the adrenal gland through the hypothalamic-pituitary-adrenal axis and lower-spinal cord sympathetic pathway activation and by the inotropic drugs given to the patient. In addition to the increased blood stream catecholamine
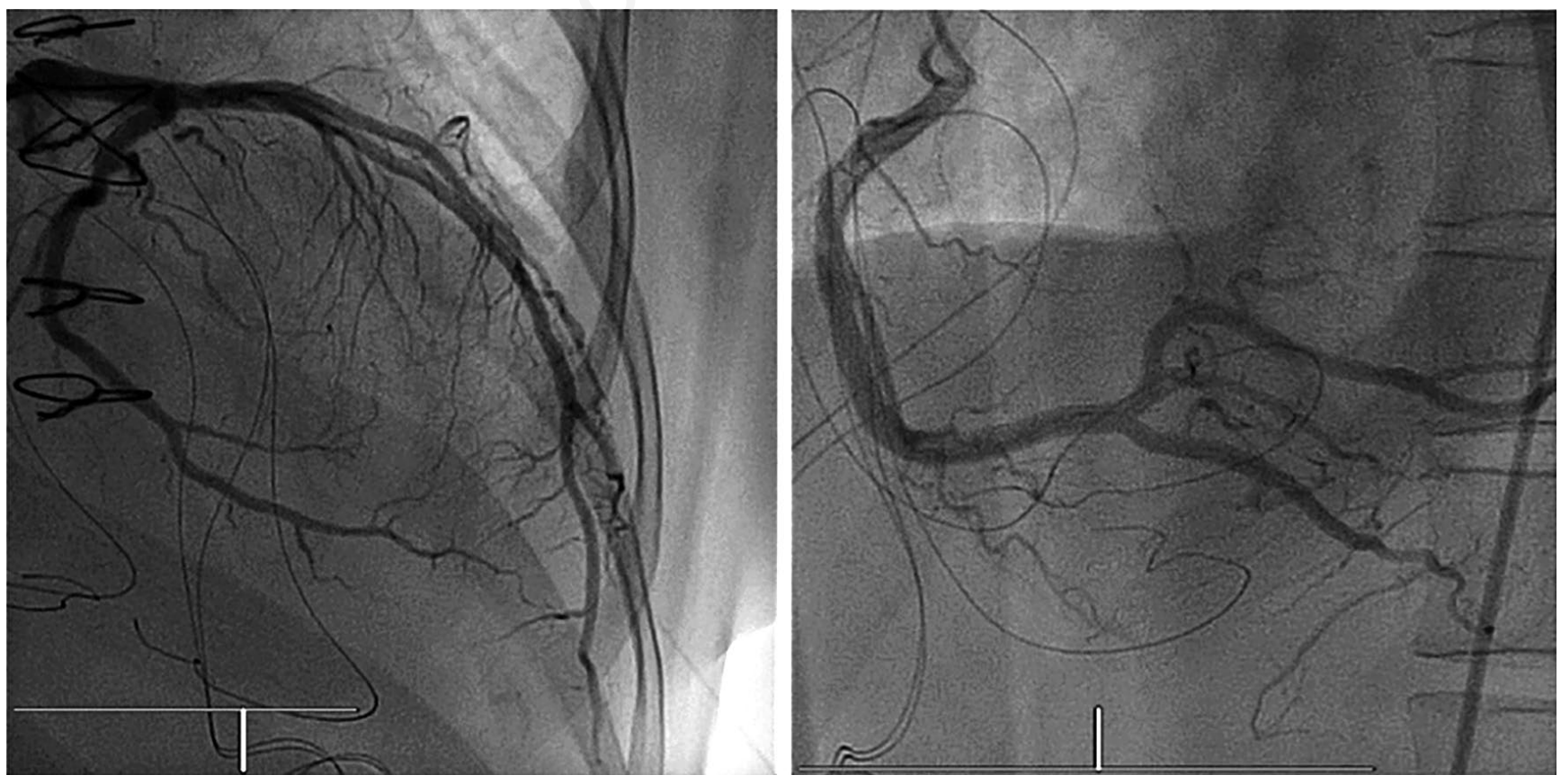

Figure 1. Coronary angiogram showing normal coronary arteries. 
concentration, also a higher sensitivity to catecholamines by the denervated heart might play a role and most likely also a decreased re-uptake capacity from the pre-synaptic neural endings. This patient had the involvement of the basal segments of both ventricles, which goes along with the younger age [13] and the epineph- rine sensitivity after the heart denervation [14]. Although there was the involvement of the right ventricle, the patient had no intra or extra hospital complications related to TTS even though the prognosis of patients with the right ventricle involvement is reported to be worse [15].

\section{First Echo}
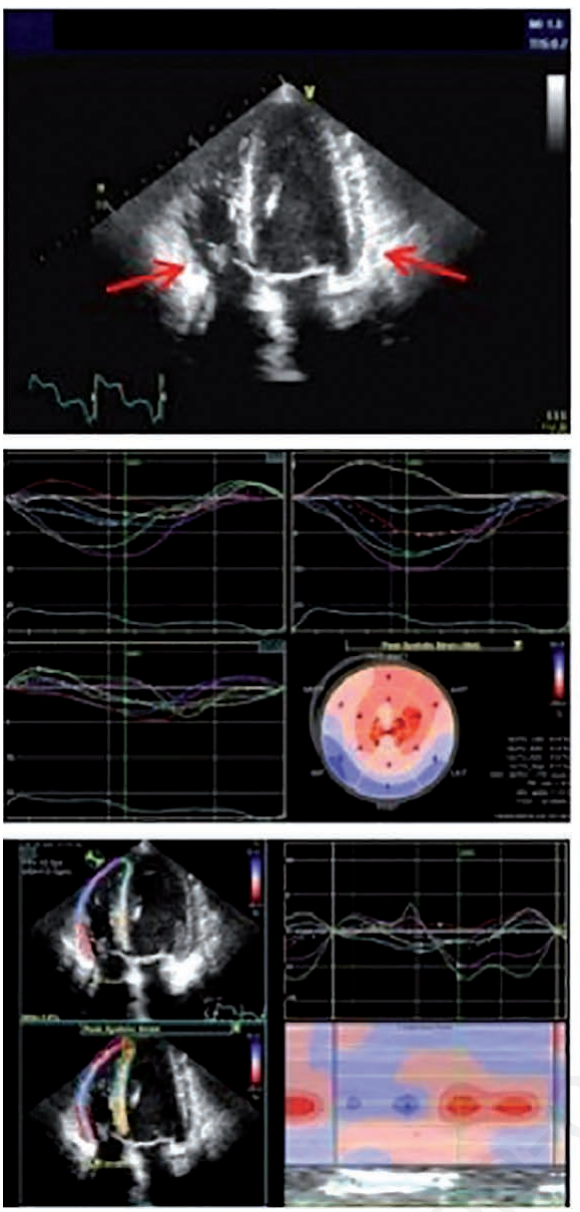

Second Echo
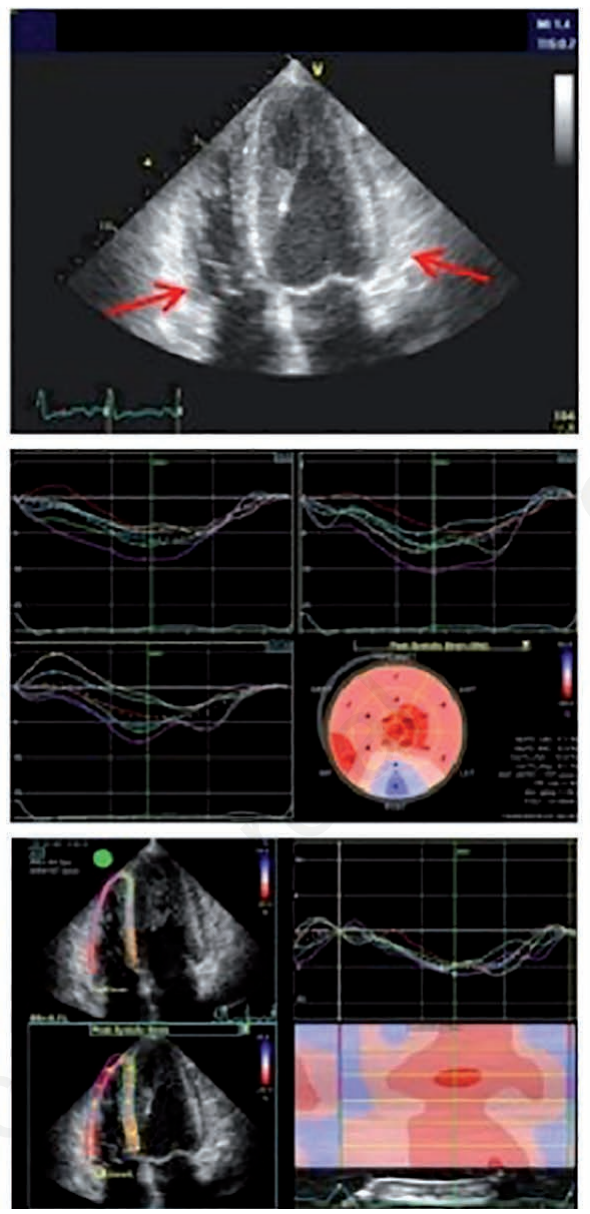

Third Echo
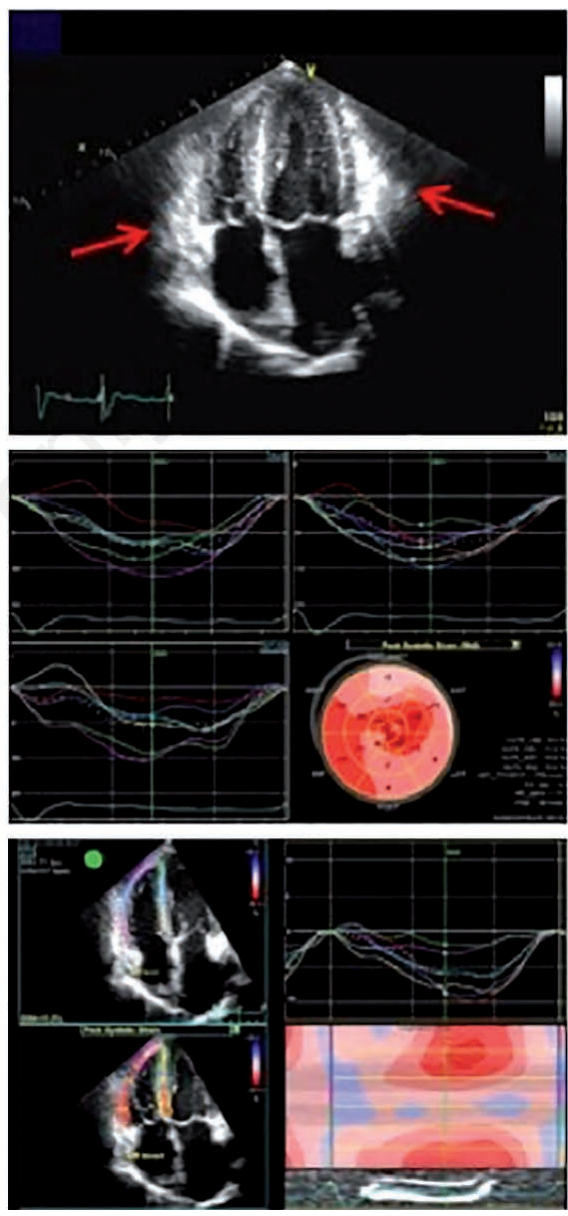

Figure 2. Echocardiogram in $4 \mathrm{CH}$ view at day 1,7 and 21 showing the regional involvement of the basal segments of the $\mathrm{LV}$ and $\mathrm{RV}$. The 2D strain of the LV and RV at day 1, 7 and 21 is represented.

Table 1. Reports of TTS peri or post HTx in literature.

\begin{tabular}{|c|c|c|c|c|c|c|c|}
\hline Author & Sex & Age & Type of TTS & Immunosuppression Rx & Time after HTx & Possible triggers & Time of recovery \\
\hline Behnes (2018) [8] & Male & 64 & Biventricular & $\begin{array}{l}\text { Cyclosporine, } \\
\text { Mycophenolate mofetil, } \\
\text { and low-dose steroids }\end{array}$ & After 9 years & $\begin{array}{l}1 \text { week after PCI } \\
\text { to lcx for NSTEMI }\end{array}$ & 10 days \\
\hline Chinali (2018) [7] & Female & 21 & Apical left ventricle & Not mentioned & After 10 years & After angry debate & 20 days \\
\hline Gastwirth (2009) [10] & Female & 55 & Apical left ventricle & $\begin{array}{l}\text { Tacrolimus, Sirolimus } \\
\text { and Azathioprine }\end{array}$ & After 1 year & $\begin{array}{c}\text { After dobutamine } \\
\text { stress echocardiography }\end{array}$ & 5 days \\
\hline Cunanan (2013) [9] & Male & 69 & Apical left ventricle & Tacrolimus & 6 years & $\begin{array}{l}\text { Hypotension after total } \\
\text { shoulder arthroplasty }\end{array}$ & 10 days \\
\hline Ravi (2018) [12] & Female (donor) & 17 & Apical left ventricle & - & Donor Peri HTx & $\begin{array}{c}\text { Motor vehicle } \\
\text { accident }\end{array}$ & 3 days \\
\hline Redfors (2015) [11] & Male (donor) & 50 & Apical left ventricle & - & Donor Peri HTx & $\begin{array}{l}\text { Subarachnoid } \\
\text { hemorrhage }\end{array}$ & 30 days \\
\hline
\end{tabular}




\section{Conclusions}

This is a rare case of TTS that happened within the first 24 hours after heart transplantation, when the heart is completely denervated. We hypothesized that the present case of TTS was related to the drug inotropic support and the endogenous catecholamines released by the adrenal medulla as response to stressful clinical setting.

\section{References}

1. Bybee KA, Kara T, Prasad A, et al. Systematic review: transient left ventricular apical ballooning: a syndrome that mimics ST-segment elevation myocardial infarction. Ann Intern Med 2004; 141:858-65.

2. Prasad A, Lerman A, Rihal CS. Apical ballooning syndrome (Tako-Tsubo or stress cardiomyopathy): a mimic of acute myocardial infarction. Am Heart J 2008;155:408-17.

3. Wittstein IS, Thiemann DR, Lima JA, et al. Neurohumoral features of myocardial stunning due to sudden emotional stress. $\mathrm{N}$ Engl J Med 2005;352:539-48.

4. Paur H, Wright PT, Sikkel MB, et al. High levels of circulating epinephrine trigger apical cardiodepression in a $\beta 2$-adrenergic receptor/Gi-dependent manner: a new model of takotsubo cardiomyopathy. Circulation 2012;126:697-06.

5. Ghadri JR, Wittstein IS, Prasad A, et al. International expert consensus document on takotsubo syndrome (Part I): clinical characteristics, diagnostic criteria, and pathophysiology. Eur Heart J 2018;39:2032-6.
6. Gordan R, Gwathmey KJ, Xie LH. Autonomic and endocrine control of cardiovascular function. World $\mathrm{J}$ Cardiol 2015;7:204-4.

7. Chinali M, Formigari R, Grutter G. Takotsubo cardiomyopathy in a young adult with transplanted heart: what happened to denervation? ESC Heart Failure 2018;5:197-200.

8. Behnes M, Baumann S, Borggrefe M, Haghi D. Biventricular takotsubo cardiomyopathy in a heart transplant recipient. Circulation 2013;128: e62-3.

9. Cunanan V, Ganesh S, Kleisli T, et al. Takotsubo cardiomyopathy in heart transplant recipient. Chest 2013;144:124A.

10. Gastwirth VG, Yang HS, Steidley DE, et al. Dobutamine stress-induced cardiomyopathy in an orthotopic heart transplant patient. J Heart Lung Transplant 2009;28:968-70.

11. Redfors T, Ramunddal J, Oras K et al. Successful heart transplantation from donor with takotsubo syndrome. Int J Cardiol 2015;195: 82-4.

12. Ravi Y, Campagna R, Rosas PC, et al. Successful heart transplantation using a donor heart afflicted by takotsubo cardiomyopathy. Proc (Bayl Univ Med Cent) 2016;29:73-4.

13. Bertin N, Brosolo G, Antonini-Canterin F, et al. Takotsubo syndrome in young fertile women. Acta Cardiol 2019:1-9. https://doi.org/10.1080/00015385. [Epub ahead of print].

14. Sarcon A, Ghadri JR, Wong G, et al. Takotsubo cardiomyopathy associated with opiate withdrawal. QJM 2014;107: 301-2.

15. Citro R, Bossone E, Parodi G, et al. "Takotsubo Italian network" Investigators (see Appendix). Clinical profile and inhospital outcome of Caucasian patients with takotsubo syndrome and right ventricular involvement. Int $\mathrm{J}$ Cardiol 2016:219:455-69. 